\title{
PERTINENCIA DE LOS INSTRUMENTOS DE DESARROLLO EMPRESARIAL OFERTADOS POR ENTIDADES QUE CONFORMAN LA COMISIÓN REGIONAL DE COMPETITIVIDAD E INNOVACIÓN DEL DEPARTAMENTO DEL QUINDÍO
}

\begin{abstract}
RELEVANCE OF THE INSTRUMENTS OF BUSINESS DEVELOPMENT OFFERED BY ENTITIES THAT CONFORM THE REGIONAL COMMISSION OF COMPETITIVENESS AND INNOVATION OF THE DEPARTMENT OF QUINDÍO
\end{abstract}

\begin{abstract}
RELEVÂNCIA DOS INSTRUMENTOS DE DESENVOLVIMENTO DE NEGÓCIOS OFERECIDOS PELAS ENTIDADES QUE CONFORMAM A COMISSÃO REGIONAL DE COMPETITIVIDADE E INOVAÇÃO DO DEPARTAMENTO DE QUINDÍO
\end{abstract}

CRUZ DIAZ-Joel, BLANDÓN LOPEZ-Alexander, CRUZ RINCÓN-Diego

Magister en Gestión del Desarrollo Regional de la Universidad Católica de Pereira. Profesor Asistente del Departamento de Economía y Finanzas de la Universidad del Tolima. Email: jhcruzd@ut.edu.co, Colombia.

PhD en Development Studies, International Institute of Social Studies of Erasmus University Rotterdam. Profesor Asociado Facultad de Ciencias Económicas y Administrativas, Universidad Del Tolima. Email. ablandonl@ ut.edu.co, Colombia

Magister en Ciencias Sociales de la Universidad Autónoma de Zacatecas, México. Becario CLACSO-CONACYT. Email: diegofcruzr@gmail.com, Colombia.

Recibido: 25 de septiembre de 2017 Aprobación definitiva: 15 de mayo de 2018 DOI: http://dx.doi.org/10.22267/rtend.181901.87 


\section{RESUMEN}

El abordaje de la pertinencia de los instrumentos de desarrollo empresarial ofertados por las entidades que conforman la Comisión Regional de Competitividad e Innovación del Quindío, se hace con base en tres corrientes teóricas de la literatura académica: teorías y políticas de desarrollo local y regional, estrategias de competitividad y análisis de cadena de valor y clústeres. En el análisis de la información obtenida se pudo determinar que se están realizando ejercicios participativos de planificación estratégica con miras a fortalecer las capacidades competitivas de la estructura empresarial, pero la oferta de apoyos no tiene en cuenta la etapa del ciclo de vida de las empresas ni las especificidades del modelo de negocio donde compiten. También se evidencia la desarticulación interinstitucional y se refleja tímidamente en algunos sectores el enfoque orientado a incentivar interrelaciones entre empresas para estimular, fortalecer y desarrollar las capacidades competitivas. Finalmente es necesario crear un sistema territorial de información para estudiar las cadenas productivas y obtener datos estadísticos para diseñar las políticas de desarrollo productivo regional.

Palabras clave: Desarrollo regional, Clúster, Cadenas de valor, Competitividad

JEL: Q13, O25, O31, R58

\section{ABSTRACT}

The approach of the relevance of the instruments of business development offered by the entities that form the Regional Commission for Competitiveness and Innovation of Quindío, is based on three theoretical currents of academic literature: local and regional development theories and policies, strategies competitiveness and value chain analysis and clusters. In the analysis of the information obtained it was possible to determine that participatory strategic planning exercises are being carried out with a view to strengthening the competitive capacities of the business structure, but the offer of support does not take into account the stage of the life cycle of the companies or the specificities of the business model where they compete. The interinstitutional disarticulation is also evidenced and 
the focus on encouraging interrelationships between companies to stimulate, strengthen and develop competitive capacities is timidly reflected in some sectors. Finally, it is necessary to create a territorial information system to study the productive chains and obtain statistical data to design policies for regional productive development.

Keywords: Regional development, cluster, value chains, competitiveness

JEL: Q13, O25, O31, R58

\section{RESUMO}

A abordagem da relevância dos instrumentos de desenvolvimento empresarial oferecidos pelas entidades que formam a Comissão Regional de Competitividade e Inovação do Quindío, é baseada em três correntes teóricas da literatura acadêmica: teorias e políticas de desenvolvimento local e regional, estratégias de competitividade e análise da cadeia de valor e clusters. $\mathrm{Na}$ análise das informações obtidas, foi possível determinar que estão sendo feitos exercícios participativos de planejamento estratégico com o objetivo de fortalecer as capacidades competitivas da estrutura do negócio, mas a oferta de suporte não leva em consideração a etapa do ciclo de vida das empresas ou as especificidades do modelo de negócios onde elas competem. A desarticulação interinstitucional também é evidenciada, embora é possível ver timidamente a intenção de alguns setores de incentivar as inter-relações entre as empresas para estimular, fortalecer e desenvolver as capacidades competitivas. Finalmente, é necessário criar um sistema de informação territorial para estudar as cadeias produtivas e obter dados estatísticos para elaborar políticas de desenvolvimento produtivo regional.

Palavras-chave: Desenvolvimento regional, Cluster, Cadeias de valor, Competitividade.

JEL: Q13, O25, O31, R58 


\section{INTRODUCCIÓN}

En Colombia se viene promoviendo desde finales del siglo XX, el enfoque de diseñar políticas y estrategias territoriales que se orientan a incentivar la generación de interrelaciones entre empresas para transformar y desarrollar las capacidades competitivas de los sistemas productivos regionales, con el objetivo de ofertar sus productos en las cadenas globales de valor en donde los consumidores son cada vez más exigentes. Desde esta perspectiva se reconoce que la atención de la política industrial y sus respectivos instrumentos de apoyo empresarial se debe centrar sobre grupos de empresas (clúster), debido a que se generan economías de escala, coordinación y eficiencia en la implementación de acciones.

En un clúster interactúan sistémicamente las empresas, el gobierno, la comunidad científica y educativa, las instituciones financieras y las demás instituciones de apoyo con sus respectivos instrumentos, con el fin de incentivar la articulación empresarial y productiva para generar ventajas para las empresas en materia de productividad e incentivos a la eficiencia, la innovación y el emprendimiento. Lo que se pretende es que los actores del desarrollo regional y local, generen un diálogo inteligente y constructivo para identificar oportunidades comunes y proveer una guía para las políticas públicas. Este enfoque se puede enmarcar dentro de las teorías del Desarrollo Económico Local (DEL), el cual según Pérez (2000) se define como:

Aquel proceso reactivador de la economía y dinamizador de la sociedad local, que mediante el aprovechamiento de los recursos endógenos existentes es capaz de estimular y fomentar su crecimiento económico, crear empleo, renta y riqueza, y sobre todo mejorar la calidad de vida y el bienestar social de la comunidad local (p.48).

El DEL proyecta objetivos como el impulso a las actividades de fortalecimiento empresarial y productivo, la organización de redes locales entre actores públicos y privados para promover la innovación productiva y empresarial, la exploración de nuevas fuentes de empleo e ingresos, la promoción de actividades de desarrollo científico y tecnológico y el diseño de instrumentos locales de desarrollo empresarial. Estas iniciativas 
contienen elementos básicos para el logro de sus propósitos los cuales deben ser conocidos y potenciados por los actores del territorio, para crear sinergias suficientes y la confluencia de los objetivos de los actores y agentes económicos (Alburquerque, 2004).

Con base en los conceptos antes mencionados, se pueden comprender los esfuerzos que se vienen realizando en las regiones colombianas desde los años noventa del siglo pasado, con el objetivo de reforzar la competitividad de las industrias estratégicas identificadas en los ejercicios de planificación territorial y que presentan requerimientos de innovación del sistema productivo local. Lo anterior obliga a las instituciones de apoyo públicas y privadas a realizar cambios sustanciales en su oferta territorial de instrumentos de desarrollo empresarial, debido a que implica tener una permanente capacidad de observación de las necesidades reales y potenciales de los empresarios y de las características del mercado de trabajo. El éxito de estas iniciativas se logra cuando los acuerdos se institucionalizan con la participación y compromiso real de los actores territoriales, porque se requiere que las acciones definidas se incorporen formalmente en los planes y programas de cada uno de los actores y agentes que promueven las transformaciones productivas.

Los instrumentos de desarrollo empresarial son herramientas que proveen instituciones públicas, privadas y académicas que se dirigen para atender necesidades de las empresas en todas las etapas de su ciclo de vida y en cualquiera de sus áreas de operación, proporcionándose a través de ofertas institucionales directas por medio de proyectos, asesorías, asistencias técnicas, capacitaciones, transferencia de tecnología, conformación de redes, ruedas de negocios, servicios de investigación, misiones comerciales, entre otras. Estos instrumentos son un servicio fundamental para el fortalecimiento de la estructura empresarial y dependen de las características económicas y sociales de las regiones, empresas y del grado de articulación de éstas con las cadenas de valor. Si bien estos factores hacen que la oferta varíe de un territorio a otro, se han identificado estrategias en diferentes experiencias estudiadas, las cuales tienen en cuenta aspectos clave como la existencia de mecanismos de armonización de oferta y demanda de dichos instrumentos, el desarrollo de estrategias de divulgación y accesibilidad, 
el establecimiento de mecanismos de generación de conocimiento en la base empresarial sobre la existencia y uso de estos servicios, además de experiencias en evaluación e impacto de estas políticas.

El Ministerio de Comercio, Industria y Turismo (MINCIT) viene promoviendo programas de refuerzo a la competitividad empresarial, para potenciar las economías regionales y locales mediante el fortalecimiento de iniciativas clústers y como parte de este ejercicio, desde el año 2008 cada departamento ha formulado su respectivo Plan Regional de Competitividad, identificando y priorizando las apuestas productivas sobre las cuales debe focalizar los instrumentos de apoyo al desarrollo empresarial. Sin embargo, según un estudio realizado por Propaís (2012) para el MINCIT, en Colombia existe una oferta de instrumentos de desarrollo empresarial con falencias en su estructuración, debido a que se diseñan sin conocer en detalle el conjunto de la oferta local y regional. Se desconoce quién ofrece ¿qué?, ¿dónde?, ¿cómo?, ¿cuánto?, las fortalezas y debilidades de los oferentes, los vacíos y la sobreoferta existente, lo cual dificulta la definición de complementariedades, coberturas, sinergias y la especialización. Por el lado de la demanda también hay falencias por el desconocimiento de su uso, la percepción de satisfacción frente a los intereses, perfiles y requerimientos de los empresarios.

Con base en un diagnóstico realizado en el año 2006 denominado la Agenda Interna, en el marco del Plan Nacional de Desarrollo 2006-2010 y el Plan Departamental 2008-2011, en el Quindío se conformó en el año 2009 la Comisión Regional de Competitividad con su respectivo Plan y una visión competitiva del Quindío para el año 2032, la cual plantea un departamento ambientalmente sostenible, sustentable, equitativo, justo e incluyente socialmente y competitivo, basado en el aumento de la diversificación de la productividad agro exportadora, un turismo y otros servicios con alto valor.

El logro de esta visión se enmarca en los objetivos estratégicos definidos para desarrollar la economía con un enfoque hacia mercados internacionales, fortaleciendo los productos y servicios de comercialización nacional, diseñando las estrategias necesarias para la generación de empresas y empleos que permitan consolidar los sectores o industrias 
priorizadas por su potencial productivo, para lo cual se requieren acciones concertadas y articuladas entre las instituciones públicas y privadas que conforman la Comisión, al igual que la participación activa y el compromiso de los empresarios. En el año 2014 la Comisión Regional de Competitividad e Innovación del Departamento del Quindío (CRCIQ) ejecutó un proyecto que buscaba fortalecer sus capacidades de intervención, mediante la identificación y caracterización de la oferta de instrumentos de desarrollo empresarial por parte de las instituciones que la conforman.

\section{FUNDAMENTO TEÓRICO}

La investigación desarrolla sus conceptos bajo tres corrientes teóricas de literatura académica: teorías y políticas de desarrollo local y regional, estrategia de competitividad y análisis de cadena de valor y clústeres. Con respecto al Desarrollo local y políticas de competitividad, según Baud (2002, p.4) "En el debate de la competitividad internacional, las capacidades de los actores locales para crear un clima favorable están ganando prioridad" y este argumento está respaldado por la atención puesta por académicos como Helmsing (2001) a la importancia de las economías externas, el aprendizaje colectivo y la calidad de la gobernanza en el fortalecimiento del desarrollo económico. En el mismo sentido, Westen (2002, p.58) sostiene que "una forma apropiada de promover el desarrollo económico local, aparte de situaciones extremas como la colusión entre los poseedores de poder político e intereses económicos, es un "anclaje político moderado de la economía", donde el estado en colaboración con el sector privado y las organizaciones de la sociedad civil, puede crear un marco conducente al crecimiento".

Por su parte Knorringa (2002, p.74) destaca el papel de los hacedores de políticas públicas en el sentido de que "debería tener como objetivo facilitar la captura de mayores etapas de valor agregado en la cadena de valor, apoyar los intentos de adquirir capacidades tecnológicas innovadoras y especialmente facilitar plataformas para permitir que los conflictos internos sean resueltos". Por último, es importante resaltar que las tendencias cada vez mayores hacia la cooperación entre actores públicos y privados en el plano local/regional trascienden la cuestión 
de la cooperación entre empresas y subrayan la necesidad de avanzar y consolidar esquemas de cooperación público/privada. La ruta de la cooperación no es fácil y presenta tensiones dadas las diferentes tipos de intereses de las empresas, las élites locales y regionales y los funcionarios gubernamentales. Según Helmsing (2000, p.97) "las acciones de promoción de la cooperación público privada se centran en la creación de meso instituciones a nivel de territorio e industria".

Con respecto a las estrategias de competitividad se presenta una revisión del cambio en el pensamiento y enfoques hacia el estudio de la competitividad industrial y las contribuciones de Michael H. Best (1990), Klaus Esser et al. (1996), J. Meyer-Stamer (1998) y Michael Porter (1990, $1998,2003)$, prestando especial atención a los elementos que contribuyen a la elaboración teórica del presente estudio. La competitividad es un concepto ampliamente conocido, aunque no existe un consenso claro sobre su significado, a pesar de que tiene implicaciones primordiales para el destino de los empresarios, especialmente en los países en desarrollo y para el sustento de sus habitantes. "La competitividad sigue siendo un concepto no bien entendido, a pesar de la amplia aceptación de su importancia" (Porter 2003: 25, la traducción es nuestra).

El entorno empresarial y los procesos de reestructuración económica mundial se condensan en gran parte desde el punto de vista teórico en el concepto de nueva competencia, el cual constituye un avance importante en la comprensión de lo que está sucediendo a nivel regional como respuesta a los principales acontecimientos que tienen lugar a nivel mundial. La teoría de Best da un papel supremo a la firma emprendedora cuya definición tiene influencia teórica sobre los conceptos Schumpeterianos de emprendimiento y competencia. Para ser competitivos, los países y las empresas tienen que congregar a varios actores en torno a objetivos de competitividad con la participación vital del gobierno, las instituciones del sector y las empresas, no a través de una política industrial tradicional, sino a través de esquemas que implican alianzas estratégicas.

Desde el punto de vista teórico, la nueva política de competitividad industrial se volvió más sistemática tras la publicación del libro de Michael 
Porter, la ventaja competitiva de las naciones (1990). Desde la perspectiva estratégica, argumenta que la competitividad es creada y no derivada de las ventajas comparativas estáticas como se aceptó tradicionalmente. La competitividad se considera como la eficiencia con la que una nación utiliza sus recursos económicos y está "arraigada en los fundamentos microeconómicos de una nación, que se manifiesta en la sofisticación de sus empresas y en la calidad de su entorno microeconómico empresarial" (Porter 2003, p.41). La innovación es un componente clave que influye en la construcción de la competitividad de una nación y apoya el esfuerzo de su industria para actualizarse (1998, p.155). Porter (2003) afirma que "las empresas de una nación deben mejorar sus formas de competir si se espera un desarrollo económico exitoso. Las empresas de una nación deben pasar de competir en ventajas comparativas (mano de obra barata o recursos naturales) a competir en ventajas competitivas derivadas de productos y procesos únicos"(p.25, la traducción es nuestra).

Con respecto a los clústeres, Porter (1998) pone especial atención a su estudio y los define como aglomeraciones geográficas de empresas, sus proveedores, las industrias relacionadas e instituciones especializadas en un campo particular en una nación, estado o ciudad (p.7). El alcance geográfico de un clúster puede variar desde una sola ciudad o estado a un país o incluso a una red de países vecinos. La importancia de los clústeres para la competitividad es que la influencian en tres formas diferentes: incrementando la productividad de las firmas o industrias constitutivas, incrementando la capacidad de innovación y por lo tanto para el crecimiento de la productividad y estimulando la formación de nuevos negocios que apoyan la innovación y expanden el clúster (Porter, 2003, p.26). En esta línea de pensamiento:

La competitividad industrial no se produce espontáneamente a través de un cambio en el marco macro o simplemente a través del emprendimiento a nivel micro. Es más bien el resultado de un patrón de interacción compleja y dinámica entre gobiernos, empresas, instituciones intermedias y la capacidad organizativa de una sociedad "(Esser et al 1996, p.27, la traducción es nuestra)

Finalmente, el análisis de las cadenas de valor proporciona un marco analítico para descifrar los resultados del desarrollo de la acción colectiva y privada de este tipo de relaciones a nivel internacional, nacional, regional 
y local. Esas contribuciones son el punto de partida para llevar a cabo el análisis de la estrategia de competitividad y apoyar el énfasis que se hace en esta investigación en los niveles meso-micro. Es importante reconocer que las regiones se convierten en actores clave cuando toman lugar en el territorio aspectos importantes tales como la capacidad de aprendizaje, la asociatividad y prácticas de redes entre empresas y organizaciones de gobernanza (Cook and Morgan 2000: 59). Según Blandón (2012), tomando en cuenta que el estado nacional promulga y provee el marco de políticas para las cadenas de valor, es importante indagar sobre el papel del estado regional en la implementación y resultados del desarrollo de acuerdos públicos/privados para la promoción del desarrollo de las mismas cadenas. Las características regionales específicas en las que se incrustan las cadenas de valor desempeñan un papel complementario, impidiendo o apoyando las intervenciones y sus resultados a nivel de cadena de valor. El anclaje regional de las cadenas de valor presupone la interacción entre sus agentes y el papel constructivo de las instituciones en la definición y resultados de los acuerdos.

\section{MATERIALES Y MÉTODOS}

Con base en los objetivos del Plan Regional de Competitividad del Departamento del Quindío (2009), es necesario fortalecer los procesos de participación de los actores comprometidos con la definición y ejecución de las acciones estratégicas requeridas para lograr su visión, por lo que se hace necesario desarrollar acciones concertadas y articuladas entre los actores comprometidos con la competitividad, la productividad y la innovación en el territorio. Uno de los requerimientos es conocer, caracterizar y analizar la pertinencia de la oferta de instrumentos de desarrollo empresarial que las instituciones de apoyo tienen disponibles para los empresarios de las apuestas productivas identificadas y priorizadas en el Plan. La oferta de servicios que contribuyen al desarrollo empresarial territorial se realiza usualmente de manera diferenciada por organizaciones que actúan de forma independiente, evidenciando situaciones como desconocimiento sobre lo que cada organización ofrece, diseño de servicios ignorando la oferta regional y nacional de otras entidades, además de una desarticulación al momento de gestionar sus servicios. De otra parte, por lo general se desconocen las necesidades 
específicas de apoyo al desarrollo empresarial de los diferentes sectores económicos, poniendo en duda la pertinencia de su oferta.

Esta investigación se presenta como un estudio de tipo descriptivo con un enfoque cualitativo y se orienta a obtener resultados que permitan identificar alternativas y propuestas a los problemas relacionados con la competitividad empresarial del Departamento del Quindío. El Método es inductivo debido a que se analizaron los casos específicos de las instituciones que hacen parte del Sistema Regional de Competitividad del Departamento, para luego concluir sobre la pertinencia de la oferta de instrumentos de apoyo a los empresarios.

La investigación es aplicada porque el objetivo es tratar de resolver un problema práctico, relacionado con la pertinencia de la oferta de instrumentos de desarrollo empresarial en el Departamento del Quindío y se centra en proponer alternativas y rutas para transformar la situación estudiada, es decir, no coloca su énfasis en lo teórico y trata de vincular la investigación con la práctica a través de la aplicación orientada en la toma de decisiones.

La población objeto de estudio fueron las instituciones que ofertan instrumentos de desarrollo empresarial y conforman el Sistema Regional de Competitividad del Quindío y para recolectar la información se realizaron entrevistas a los responsables de las instituciones oferentes, para lo cual se diseñaron los instrumentos respectivos con los ítems necesarios y suficientes para caracterizar la oferta de instrumentos de desarrollo empresarial. También se realizó revisión bibliográfica y documental pertinente sobre el tema, para complementar y contrastar la información.

Con base en la conformación vigente de la CRCIQ (Comisión Regional de Competitivdad e innovación del Quindío) y de acuerdo con el análisis realizado por parte de su Secretaría Técnica, se determina que sólo las siguientes instituciones tienen oferta de instrumentos de apoyo: 


\begin{tabular}{|l|l|}
\hline Sector Público \\
\hline - & Gobernación del Departamento del Quindío \\
& Secretaría de Turismo, Industria y Comercio, Secretaría de \\
& Planeación y la Secretaría de Agricultura, Desarrollo Rural y \\
& Medio Ambiente. \\
- & Alcaldía Municipal de Armenia - Secretaría de Desarrollo \\
& Económico. \\
- SENA Regional Quindío \\
- Corporación Autónoma Regional Quindío (CRQ) \\
\hline Sector Productivo \\
\hline - Cámara de Comercio de Armenia y del Quindío. \\
- Cotelco - Capítulo Quindío \\
\hline Sector Académico \\
\hline - Universidad del Quindío. \\
- Universidad La Gran Colombia. \\
\hline
\end{tabular}

\section{RESULTADOS Y DISCUSIÓN}

La tabla No. 1 se obtuvo del documento final del proyecto denominado "Fortalecimiento de la Comisión Regional de Competitividad e Innovación del Quindío" y muestra la cantidad de instrumentos de desarrollo empresarial que cada una de las entidades ofrece a los empresarios de los diferentes sectores productivos del Departamento del Quindío. Además describe las actividades de apoyo que atiende y se pudo evidenciar que en orden de importancia, priman las actividades relacionadas con las asesorías específicas, luego viene la asistencia técnica, la formación, el emprendimiento, la formalización, el apoyo comercial y finalmente los apoyos financieros. También se puede inferir que los instrumentos ofertados por las entidades del sector público representan el $81 \%$ del total y las entidades educativas de orden superior del departamento sólo ofertan el $11 \%$. 


\section{Tabla 1}

Comisión regional de competitividad e innovación del Quindío

Oferta de instrumentos de apoyo empresarial

\begin{tabular}{|c|c|c|c|c|c|c|c|}
\hline \multicolumn{8}{|c|}{ RESUMEN } \\
\hline \multirow{2}{*}{$\begin{array}{l}\text { CANTIDAD DE } \\
\text { INSTRUMENTOS } \\
\text { OFERTADOS }\end{array}$} & \multirow[b]{2}{*}{ ENTIDAD OFERENTE } & \multicolumn{6}{|c|}{ ACTIVIDADES DE APOYO QUE EJECUTA EL INSTRUMENTO } \\
\hline & & $\begin{array}{r}\text { Asesoria } \\
\text { especifica }\end{array}$ & Formación & $\begin{array}{l}\text { Asistencia } \\
\text { técnica }\end{array}$ & Financiero & $\begin{array}{l}\text { Emprendimientoy } \\
\text { formalización }\end{array}$ & Apoyo comercial \\
\hline 18 & GOBERNACION DEL QUINDIO & 16 & 15 & 13 & 1 & 4 & 4 \\
\hline 13 & ALCALDIA DE ARMENIA & 13 & 7 & 10 & 2 & 5 & 6 \\
\hline 5 & SENA & 3 & 3 & 1 & 1 & 3 & o \\
\hline 7 & CRQ & 2 & 4 & 4 & 1 & 3 & 1 \\
\hline 43 & SECTOR PUBLICO & 34 & 29 & 28 & 5 & 15 & 11 \\
\hline 3 & CAMARA DE COMERCIO DE ARMENI & 3 & 3 & 3 & 1 & 2 & 2 \\
\hline 1 & COTELCO - CAPITULO QUINDIO & 1 & o & 1 & $\mathrm{o}$ & o & 1 \\
\hline 4 & SECTOR PRODUCTIVO & 4 & 3 & 4 & 1 & 2 & 3 \\
\hline 1 & UNIVERSIDAD DEL QUINDIO & 1 & o & $\mathrm{o}$ & $\mathrm{o}$ & 1 & $\mathrm{o}$ \\
\hline 2 & UNIVERSIDAD LA GRAN COLOMBIA & 2 & 2 & $\mathrm{o}$ & $\mathrm{o}$ & 2 & 0 \\
\hline 3 & EAM & 3 & o & 3 & 1 & 1 & 2 \\
\hline 6 & SECTOR ACADEMICO & 6 & 2 & 3 & 1 & 4 & 2 \\
\hline 53 & TOTALES & 44 & 34 & 35 & 7 & 21 & 16 \\
\hline \multirow{2}{*}{$100 \%$} & & $83,02 \%$ & $64,15 \%$ & $66,04 \%$ & $13,21 \%$ & $39,62 \%$ & $30,19 \%$ \\
\hline & & $\begin{array}{l}\text { Asesoria } \\
\text { especifica }\end{array}$ & Formación & $\begin{array}{c}\text { Asistencia } \\
\text { técnica }\end{array}$ & Financiero & $\begin{array}{l}\text { Emprendimiento } \\
\text { formalización }\end{array}$ & Apoyo comercial \\
\hline
\end{tabular}

Fuente: Grupo de Investigación Gestión del Desarrollo UGCA

Analizando en detalle el informe final de la investigación, se pudo establecer también que en la ejecución de cada uno de los instrumentos ofertados por las entidades no se hizo un proceso de articulación con las metas establecidas en el Plan Regional de Competitividad, ni obedecen a un proceso de concertación inter-institucional. Cada entidad define, diseña y ejecuta de manera aislada los instrumentos de apoyo a los empresarios, lo cual no permite canalizar las acciones y los recursos de una manera sistémica hacia el logro de los objetivos territoriales propuestos.

Las entidades del sector público como la Gobernación del Quindío, La Alcaldía de Armenia, el SENA (Servicio Nacional de Aprendizaje) y la $\mathrm{CRQ}$ (Corporación Autónoma regional del Quindío) se caracterizan por ofertar los instrumentos de desarrollo empresarial hacia los sectores: Agropecuario, Agroindustria Alimentaria, Turismo, Servicios logísticos, Software, Educación y emprendimiento para la competitividad, Marroquinería, Manufacturas, Comercio, Construcción, Muebles, 
Transporte, Confecciones, Servicios Inmobiliarios, Artesanías, Industria, Guadua, Frutas y Hortalizas.

Las entidades del sector académico y productivo como la Cámara de Comercio de Armenia, COTELCO (Asociación Hotelera y Turística de Colombia) y las Universidades se caracterizan por ofertar los instrumentos que promueven el desarrollo empresarial, el emprendimiento, la formalización y la competitividad en las empresas de los sectores mencionados anteriormente, evidenciando los esfuerzos de todas las entidades que componen la CRCIQ (Comisión Regional de Competitividad e Innovación del Quindío), pero igualmente es evidente el bajo o inexistente nivel de articulación de procesos y acciones para gestionar la competitividad empresarial de la región.

Con respecto a los recursos aplicados por las entidades en los diferentes instrumentos de apoyo empresarial, la Gráfica 1 permite establecer que en los años 2012 y 2013, el sector público financió aproximadamente las tres cuartas partes de los recursos destinados al desarrollo empresarial regional. Un cuarto de la financiación corrió por cuenta de las entidades del sector productivo y académico. El valor total destinado por las entidades que componen la CRCIQ (Comisión Regional de Competitividad e Innovación del Quindío) a financiar los instrumentos de apoyo fue de $\$ 67.986 .394 .892$, lo cual corresponde a una cifra importante que requiere la existencia de mecanismos de evaluación del impacto de las acciones ejecutadas.

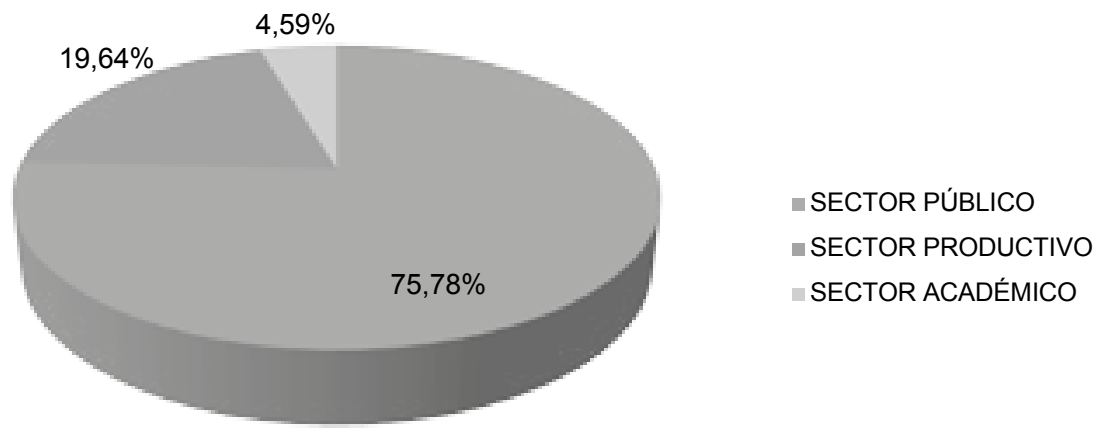

\section{Figura 1}

Financiación de los instrumentos de apoyo empresarial por sectores

Fuente: Grupo de Investigación Gestión del Desarrollo UGCA 
En la coyuntura económica que vive el Departamento del Quindío, se necesita promover estrategias para que la región, sus empresas e instituciones de apoyo sean competitivas en el corto, mediano y largo plazo, es decir, fortalecer sus procesos de articulación, planificación y ejecución de las acciones, mediante la implementación de un enfoque de gobernanza interinstitucional para la $\mathrm{CRCIQ}$. Esta gobernanza para la competitividad regional establece que la sociedad en un determinado territorio, no se rige únicamente por el gobierno, sino que éste es parte de una red compleja de interacciones entre instituciones y grupos, por lo tanto, se trata de distribuir el poder político y socioeconómico a través de una red o sistema complejo de relaciones en el que obviamente el gobierno no es el único que planifica. Marginar algunos actores o sobre representar a otros, puede generar una crisis de gobernanza y la red pierde la capacidad de canalizar los distintos intereses.

El desafío es transitar hacia la gobernanza basada en los procesos colaborativos y abiertos en redes, entendidas estas como espacios permanentes para la colaboración público-privada, donde gobiernos, empresas y agentes de innovación interactúan reflexivamente en torno a la competitividad regional. Según Barro (1998), las regiones de mayor éxito serán las que tengan la capacidad para generar redes de empresas, e instituciones que permitan utilizar sus recursos, formación continua, sistemas tecnológicos y de comunicación, -entre otros- sistémicamente, fomentando la movilidad del capital, el recurso humano y potenciando la innovación en los emprendedores.

\section{v. CONCLUSIONES}

El liderazgo ejercido por los gobiernos locales y regionales en el sistema empresarial permite establecer y desarrollar alianzas públicoprivadas, especialmente cuando están acompañadas de compromisos políticos y la asignación de recursos dentro de un marco de políticas públicas regionales. La participación del gobierno regional es crucial para asegurar la continuidad y la efectividad del proceso de políticas para promover los clústeres y cadenas de valor porque ayuda a institucionalizar el proceso. Los recursos públicos se mejoran sistemáticamente mediante la cooperación con otras partes interesadas en los acuerdos de cooperación para promover la competitividad de las empresas bien 
sea a nivel de clúster o cadena de valor, incluidas las universidades, las empresas, las asociaciones de productores agrícolas, los gremios empresariales y los organismos descentralizados.

Dentro de este esquema, el gobierno regional debe señalar a los otros miembros de la $\mathrm{CRCIQ}$ que su coordinación o participación activa en las actividades de promoción e implementación de las políticas de desarrollo productivo (fortalecimiento de la micro, pequeña y mediana empresa mediante el esquema del clúster empresarial), no significa ninguna pérdida de independencia por parte de la Comisión. De hecho, este organismo colectivo se concibe como una red independiente y no burocrática de organizaciones del sector público y privado.

Se logra determinar que la prioridad de atención de los instrumentos de apoyo empresarial ofertados por las instituciones que conforman la CRCIQ, se enfocan hacia actividades relacionadas con las asesorías específicas, la formación y la asistencia técnica. En una menor proporción se atienden necesidades del aparato productivo regional relacionadas con emprendimiento, apoyo comercial y financiamiento.

Se pudo identificar un gran número de instrumentos de apoyo genéricos que aplican para todos los sectores y no tienen en cuenta las etapas del ciclo de vida en que se encuentran las empresas ni las especificidades del modelo de negocio donde compiten. Los instrumentos de desarrollo empresarial deben diseñarse para atender requerimientos específicos que van desde los que apoyan la incubación y nacimiento de las empresas, hasta los proyectos estratégicos de crecimiento y expansión.

También se pone de manifiesto la desarticulación interinstitucional en la oferta de instrumentos de apoyo empresarial, lo cual refleja que aún no se ha incorporado en las instituciones el enfoque de diseñar políticas y estrategias territoriales orientadas a incentivar la generación de interrelaciones entre empresas para estimular, fortalecer y desarrollar las capacidades competitivas de la estructura productiva local. Los instrumentos de apoyo así ofertados atienden sólo necesidades y requerimientos de los empresarios de una manera aislada y en muchas ocasiones se duplican los esfuerzos cuando varias instituciones atienden 
las mismas necesidades. La desarticulación institucional e instrumental identificada, debe servir como elemento de reflexión para que la $\mathrm{CRCIQ}$ asuma el liderazgo y se convierta en el escenario legítimo para diseñar y promover los mecanismos idóneos para revisar y ajustar la oferta de instrumentos de apoyo, que posibilite optimizar los recursos y atender de manera sistemática los requerimientos empresariales.

Fomentar iniciativas clúster como estrategia de articulación de la institucionalidad de apoyo al desarrollo empresarial en el Departamento del Quindío, implica profundizar en los lineamientos y las acciones propuestas con base en el Programa "Rutas Competitivas" del MINCIT para los sectores de turismo rural, cafés diferenciados y curtiembres; estos sectores han recibido el apoyo de diversas instituciones que han permitido avances importantes en el fortalecimiento de su estructura productiva y competitiva, pero falta intensificar el nivel de articulación institucional para consolidar la ejecución de los planes de acción que se han formulado.

Se evidenciaron esfuerzos en las acciones realizadas con los instrumentos de apoyo enfocados hacia sectores como el turismo, los cafés especiales y las curtiembres, como parte de la política de clúster que busca fomentar iniciativas locales para eliminar o minimizar las restricciones a la productividad de las empresas y enfatiza en la complementariedad de los eslabones entre industrias, pero es necesario inducir dicha política hacia otros sectores productivos priorizados. Esto implicará re-direccionar el papel del sector privado, del gobierno, de los gremios y de las instituciones de educación e investigación, para generar un diálogo inteligente y constructivo e identificar oportunidades comunes y proveer una guía de política pública en materia de desarrollo empresarial.

Hay sectores como el turismo de salud, fabricación de muebles, artesanías, software y tecnologías de la información, aguacate, industrialización de la guadua y construcción, que vienen formulando sus propios ejercicios de planeación estratégica y definición de líneas de acción bajo esquemas metodológicos similares al Programa Rutas Competitivas, que requieren también de la acción articulada de la 
institucionalidad que conforma la CRCIQ con sus instrumentos de apoyo empresarial. Una "Ruta Competitiva" se fundamenta en la conformación de equipos locales integrados por personal idóneo proveniente de los actores comprometidos, quienes ejecutan las fases relacionadas con la identificación de los desafíos del clúster, generación de la estrategia y lanzamiento de acciones, es decir, elaboran una hoja de ruta estructurada para fortalecer el clúster definido. Esto se realiza a través de la definición de una visión que desarrolla un programa de mejoramiento competitivo de las empresas en segmentos de negocios estratégicos, pero que además cuentan con el apoyo institucional y empresarial local.

Para el sector agropecuario no cafetero también es necesario afinar instrumentos específicos de apoyo empresarial que permitan a los productores de plátano, yuca, cítricos, piña y papaya, realizar todo el proceso requerido para incentivar la generación de interrelaciones entre empresas, su caracterización y el desarrollo de un proceso participativo de planeación estratégica, que permita identificar una hoja de ruta para desarrollar sus capacidades competitivas.

Se puede inferir que en el Departamento del Quindío se están realizando ejercicios participativos de planificación estratégica con miras a fortalecer las capacidades competitivas de la estructura empresarial, a partir de iniciativas que buscan crear y consolidar encadenamientos productivos en sectores importantes para la economía regional. El paso a seguir con todas estas iniciativas es lograr el apoyo institucional de una Comisión Regional de Competitividad e Innovación fortalecida, que posibilite la ejecución de las hojas de ruta mediante la focalización de sus instrumentos de apoyo empresarial hacia el desarrollo de las acciones allí planteadas y lograr los objetivos estratégicos de favorecer y estimular la innovación y el desarrollo competitivo.

Los instrumentos de apoyo empresarial ofertados carecen en su mayoría de la visión de clúster como un proceso estratégico, participativo y riguroso para lograr cambios económicos y sociales para la región y que orienten las empresas al mercado por medio de procesos inclusivos que involucran empresas grandes, pequeñas, proveedores, instituciones públicas y privadas. Lograr coordinar las actuaciones 
dispersas de la institucionalidad, gremios y empresarios, se convierte en un reto para la $\mathrm{CRCIQ}$, porque es necesario cambiar el paradigma del enfoque asistencialista de los apoyos empresariales, hacia un enfoque fundamentado en la innovación productiva desde la perspectiva tecnológica y de los mercados.

Los representantes legales de las instituciones que integran la CRCIQ conocían y fueron informados sobre los objetivos del proyecto relacionados con la necesidad de identificar y caracterizar la oferta de instrumentos de desarrollo empresarial, pero fue difícil y traumático para que algunas suministraran la información y facilitaran los documentos, estudios y diagnósticos; lo que pone de manifiesto la falta de compromiso en algunos casos y de celos institucionales en otros, que dificultan el desarrollo de los procesos de investigación, planificación y articulación que requieren la conformación de redes de cooperación. Se necesita un cambio de actitud y una mayor apertura por parte de las instituciones que conforman la $\mathrm{CRCIQ}$ para construir estas conexiones, las cuales demandan cambios fundamentales en el pensamiento y comportamiento de sus dirigentes.

Ninguno de los instrumentos de apoyo empresarial ofertados se enfoca a la creación de un sistema territorial de información que permita estudiar las cadenas productivas y la ubicación física de sus empresas en el territorio, para conocer y comprender las relaciones económicas de la estructura de la cadena de valor actual (Proveedores, comercializadores, clientes, instituciones de apoyo). Crear instrumentos de este tipo permite obtener datos estadísticos con variables económicas y sociales que son el soporte para diseñar políticas y planes para el desarrollo productivo regional.

Las universidades atienden los requerimientos empresariales por medio de sus centros de desarrollo y principalmente se enfocan en las asesorías específicas que brindan por intermedio de los estudiantes que cursan los últimos semestres de sus carreras, pero es necesario que los grupos de investigación se vinculen directamente con los gremios y empresarios para generar alianzas que permitan identificar y promover proyectos estratégicos regionales innovadores para impactar la estructura empresarial con visión 
competitiva. También se requiere que las universidades flexibilicen la oferta académica para formar el recurso humano capaz de responder a los requerimientos del sistema productivo regional.

Las universidades y centros de investigación regional pueden aportar al conocimiento profundo de las diferentes características del sistema económico, productivo y social local, mediante la realización de estudios específicos y esfuerzos sistemáticos y continuos de investigación, que suministren información confiable y pertinente sobre el territorio, con el fin de elaborar mapas y líneas base para las diferentes variables necesarias en los análisis que realizan los responsables de gestionar las iniciativas clúster.

Los servicios territoriales de apoyo para el desarrollo empresarial requieren de una oferta adecuada y selectiva de instrumentos de desarrollo empresarial para micro y pequeñas empresas, que brinden apoyo en materia de formación e información tecnológica, comercial, financiera, de control de calidad, innovación productiva y de procesos, de gestión y cooperación empresarial entre otros, de acuerdo con las características y condiciones específicas dentro de los segmentos empresariales y sus encadenamientos productivos. Esta oferta debe atender no solo las demandas expresadas por los empresarios, sino también las señales de los mercados estratégicos, por lo cual se expresa la necesidad de diferenciar y focalizar los instrumentos.

No existe una oferta de instrumentos de apoyo empresarial que apunte a la construcción de capital social, que fomente la cultura proactiva y emprendedora autónoma, al igual que una actitud proactiva del gobierno con relación al desarrollo productivo. La identidad regional y el capital social son el resultado de la conjunción de elementos históricos, culturales y geográficos que se van construyendo localmente a partir de la participación, el diálogo, la confianza y la concertación de los actores y agentes cuando se enfrentan a situaciones comunes.

Es necesario fortalecer los instrumentos de apoyo empresarial que permitan reconocer la importancia que tienen las micro y pequeñas empresas que son la base de la estructura productiva regional, pero igualmente admitan su fragilidad organizacional y la necesidad de 
transitar hacia modelos efectivos de gestión productiva, financiera y de mercados acordes con las dinámicas económicas, por lo cual se deben generar instrumentos específicos para el fomento de la asociatividad y el fortalecimiento de sus capacidades productivas y competitivas. Se requiere la creación de fondos locales para el fomento y fortalecimiento de los pequeños productores con criterios flexibles y esquemas de capacitación y acompañamiento técnico que permitan eliminar los obstáculos para acceder a la oferta tradicional de líneas de financiamiento.

A nivel internacional existen experiencias de armonización de la oferta de instrumentos de desarrollo empresarial que pueden servir de referencia para la $\mathrm{CRCIQ}$, entre los cuales se puede relacionar la creación de un sistema central de información y referenciación cuya estructura puede ir desde los directorios de oferentes, sistemas detallados de monitoreo de los sectores, hasta plataformas que actúan como redes sociales con servicios empresariales como lo sugiere el estudio de Propais (2012). Según Zandniapour (2004), hay experiencias sobresalientes en México, Brasil y España que unificaron plataformas de información con los servicios de apoyo empresarial para contar con información específica y ejecutar diversos programas de promoción e integración industrial que se estaban implementando. También en Tailandia, según Yokakul y Zawdie (2010), hay experiencias importantes de articulación entre universidad, empresa y estado para generar procesos de innovación en MYPES integradas al Sistema Nacional de Innovación.

En cuanto al impacto de políticas y programas de desarrollo empresarial, no existe evidencia sobre mecanismos de evaluación del impacto real de estos instrumentos y servicios. Según plantea Zandniapour (2004, $p, 45)$, "existen indicadores para evaluar los programas de desarrollo empresarial enfocados en cadenas de valor, clúster y fortalecimiento de la competitividad, los cuales se concentran en trabajar con asociaciones y agremiaciones que tienen fuerte vinculación entre sus miembros y que son un vehículo efectivo en la promoción de instrumentos de apoyo a los empresarios". 


\section{REFERENCIAS}

(1) Alburquerque, F. (2004). Desarrollo económico local y descentralización en América Latina. Revista Cepal No. 82. Santiago de Chile.

(2) Baud, I.S.A and Post J. (2002). Realigning actors in an urbanizing world. Governance and institutions from a development perspective. ASHGATE, 2002.

(3) Barro, R. (1998). Determinants of Economic Growth: A Cross-Country Empirical Study. MIT Press Books, The MIT Press, Edition 1, Volume 1, Number 0262522543, April.

(4) Best, M.H. (1990). The new competition. Institutions of industrial restructuring. Cambridge: Polity Press.

(5) Blandon, L. (2012). Economic Restructuring and Value Chains: The Search for Regional Competitiveness in Colombia. International Institute of Social Studies. EUR-ISS.

(6) Cook P and Morgan, K (1998). The associational economy: firms, regions and innovation. Oxford, Oxford University Press.

(7) CRCIQ. (2009). Plan Regional de Competitividad del Quindío. Armenia.

(8) Esser, K. Hillebrand, W. Messner, D. and Meyer-Stamer, J. (1996). Systemic competitiveness. New governance patterns for industrial development. German Development Institute, Berlin, 1996 Frank Cass. www.meyer-stamer-dc/1996/ sysco-book.pdf.

(9) Helmsing, A.H.J. (2001). Externalities, learning and governance: New perspectives on local economic development. Development and Change, Vol. 32, pp 227-308.

(10) Helmsing, A.H.J. (2000). Externalities, learning and governance perspectives on local economic development. Inaugural Address as Professor of Local Regional Development delivered on 27 January, 2000. ISS, The Hague.

(11) Meyer-Stamer, J. (1998). Clustering, Systemic competitiveness and commodity chains: How firms, business associations and government in Santa Catarina/ Brazil respond to globalization. International Institute for Labor Studies, Geneva, 09-10 March, 1998. 
(12) Pérez, B. (2000). Desarrollo Local: Manual de Uso. Editorial ESIC. Madrid, España.

(13) Porter, M. (1990). The competitive advantage of nations. New York: The Free Press.

(14) Porter, M. (1998). On Competition. Harvard Business School Press, 1998, $485 p$.

(15) Porter, M. (1998b). Clusters and the new economics of competition. Harvard Business Review 76(6): 77-90.

(16) Porter, M.E. (2003). Building the microeconomic foundation of prosperity: findings from the Microeconomic competitiveness index. The Global competitiveness Report, 2002-2003, pp.19-50. New York: Palgrave McMillan, World Economic Forum.

(17) Propaís. (2012). Diagnóstico del mercado de servicios de desarrollo empresarial en Colombia: Fortalezas, retos y oportunidades. Bogotá.

(18) Knorringa, P. (2002). Revista Innovation and small enterprises in the Third World. Pp 48-65.

(19) Westen, G. (2002) 'Embeddedness: the role of local factors in economic development'. In I. Baud and J. Post (eds), Realigning actors in an urbanizing world. Governance and institutions from a development perspective. Aldershot: Ashgate Publishers.

(20) Yokakul, N., \& Zawdie, G. (2009). The role of triple helix for promoting social capital, industrial technology and innovation in the SME sector in Thailand. Science, Technology \& Society, 14(1), 93-117.

(21) Zandniapour, L. Sebstad, J. y Snodgrass, D. (2004). Review of Impact Assessments of Selected Enterprise Development Projects. AMAP Business Development Services (BDS) Knowledge and Practice (K\&P) Research Task Order. 